\title{
AN EXPLICIT SPATIAL MODEL OF YEAST MICROCOLONY GROWTH*
}

\author{
HENRIK JÖNSSON ${ }^{\dagger}$ AND ANDRE LEVCHENKO $\ddagger$
}

\begin{abstract}
Here we present a single-cell-based model of cell-cell interactions in a growing yeast colony. We examine the influence of multiple factors on the colony morphology and switch to a foraging growth behavior. We find that the cell-growth inhibition by neighboring cells along with polar division growth patterns are the most significant factors driving the exploratory behavior of a colony.
\end{abstract}

Key words. yeast colony, cell adhesion, cell division, deterministic modeling

DOI. $10.1137 / 040603255$

1. Introduction. Fungal infections remain an important and widespread cause of human, animal, and plant disease. Annual losses of various crops to pathogenic fungi are estimated to result in a deficit of food sufficient to feed tens of millions of people (see $[7,21]$ for examples of rice infections). Yeasts are prominent causes of corneal, vaginal, and other infections in humans as well as a widespread cause of contamination in biomedical devices used in contact with human tissue, including catheters and sutures $[4,6]$. To combat these infections, it is important to understand how yeast-cell communities develop into complex and sophisticated cell ensembles possessing, among other characteristics, unexpectedly intricate internal morphology.

Although the lab strains of the commonly used baker's yeast Saccharomyces cerevisiae normally form simple round colonies devoid of any internal structure, recent wild-type isolates can form structurally complex colonies with morphological patterns present on multiple spatial scales [12]. These scales include the scale of the whole colony, at which various forms of growth-front instabilities are prominently present; the scale of large subparts of a colony, at which structures such as "spokes" and "cables" have been observed; the scale of smaller subparts of the colony, at which granular structures are often observed; and finally the scale of single cells, where patterns of cells connected together in less defined but still recognizable ensembles are present $[12,18]$. Such hierarchical organization is a wonderful example of multiscale morphological organization of developing living tissues and should be addressed in a manner utilizing a multiscale modeling approach. However, so far, the mathematical and computational models of yeast-colony formation have been restricted to the description of growth-front instabilities at the edge of the expanding colony.

The models describing the dynamics of the growing front of a developing colony are based on a concept of "blocks" of yeast cells interacting with one another in a field of decreasing nutrient concentration and, potentially, a field of secreted metabolites $[13,19]$. Although these models are far from being realistic due to the abstraction

\footnotetext{
*Received by the editors January 15, 2004; accepted for publication (in revised form) October 4, 2004; published electronically February 9, 2005.

http://www.siam.org/journals/mms/3-2/60325.html

${ }^{\dagger}$ Department of Theoretical Physics, Complex Systems Division, Lund University, Sölvegatan 14A, S-223 62, Lund, Sweden (henrik@thep.lu.se). This author's research was supported in part by the Knut and Alice Wallenberg Foundation through Swegene.

${ }^{\ddagger}$ Department of Biomedical Engineering, The Whitaker Institute for Biomedical Engineering, The Johns Hopkins University, 208C Clark Hall, 3400 N. Charles St., Baltimore, MD 21218 (alev@bmv. jhu.edu).
} 
of describing multiple interacting cells as a single block, they can, to a high degree, predict large-scale colony behavior. For instance, characteristic Eden-like patterns are a common result of such models.

As cells grow in a field of ever decreasing nutrients, their metabolic states are likely to change. In particular, it is predicted that upregulation of multiple genes, including the one coding for Flo11p, a protein responsible for cell-cell adhesion, can take place $[1,18]$. Taking the effects of cell adhesion into account, one can computationally reproduce both the shape of a colony and some of its internal morphological features, such as high granularity and cell spokes (A. Levchenko, unpublished data). However, some features remain unexplained. For example, it is not clear why certain parts of the colony display what are apparently areas of directed, parallel cell growth, large enough to involve thousands of cells. Here, we propose to extend the model description to consider the effects of differential cell-division patterns on the morphology of small parts of a colony at single-cell resolution.

An important feature of the model proposed here is an explicit accounting for the mechanical interaction between cells, leading to cell displacements and formation of nontrivial colony morphologies. This model capability is especially important since yeast cells are rather passive compared to other eukaryotic cells. Although they can undergo periods of directed growth, under normal circumstances the cells are spherical and do not actively move. Thus the origin of spatial inhomogeneities in colony growth is rather difficult to account for. Here we suggest that cell-cell adhesion and the patterns of cell division, along with more trivial cell displacements in an expanding colony, are the primary force-generating parameters. We then explore how these parameters may facilitate interesting variations in the shape and size of a growing colony.

2. Biological background and assumptions. The cells of the yeast $S$. cerevisiae can be of two types: haploid (a single set of chromosomes) and diploid (a double set of chromosomes). Both cells are round in shape (although diploid cells can be slightly elongated) and divide by budding; that is, they form a mother-daughter cell pair, with the daughter cell growing gradually from a small bud into a larger cell while attached to the mother cell throughout growth. At cell division, the nucleus of the mother cell divides, and one of the resulting separate nuclei moves into the mother cell and the other into the bud of the daughter cell, followed by the physical separation of the unevenly sized cells. The smaller-size daughter cell then continues growing before reaching the size of the mother cell and undergoing its own cell division. The mother cell can continue dividing a multiple, although not an unlimited, number of times.

The location of the bud on the surface of the mother cell can be described by a vector $\chi$ connecting the center of the mother cell and the center of the bud. Interestingly, for a given mother cell, the direction of this vector for subsequent cell divisions is not random. There are several conventional types of division pattern, which we consider in sequence $[2,5,11,14,15]$ (cf. Figure 2.1C). In the axial pattern, the vectors in the set $\chi_{i}, i=1,2, \ldots$, are almost coincident; that is, all the $\chi_{i}$ differ from one another by a small angle $\gamma_{i} \ll 1$. The bipolar pattern is more complicated in that the $\chi_{i}$ and $\chi_{i-1}$ for all $i$ are collinear but opposite in direction $\left(\gamma_{i} \cong \pi\right)$. Thus each successive cell division occurs at the opposite "pole" of the location of the mother cell. Both axial and bipolar patterns also hold when a daughter cell becomes able to produce a bud and thus become a mother cell. Here, however, another distinction is sometimes made. If the axial pattern sets for a daughter cell, so that $\chi_{i}$ is collinear 
A.
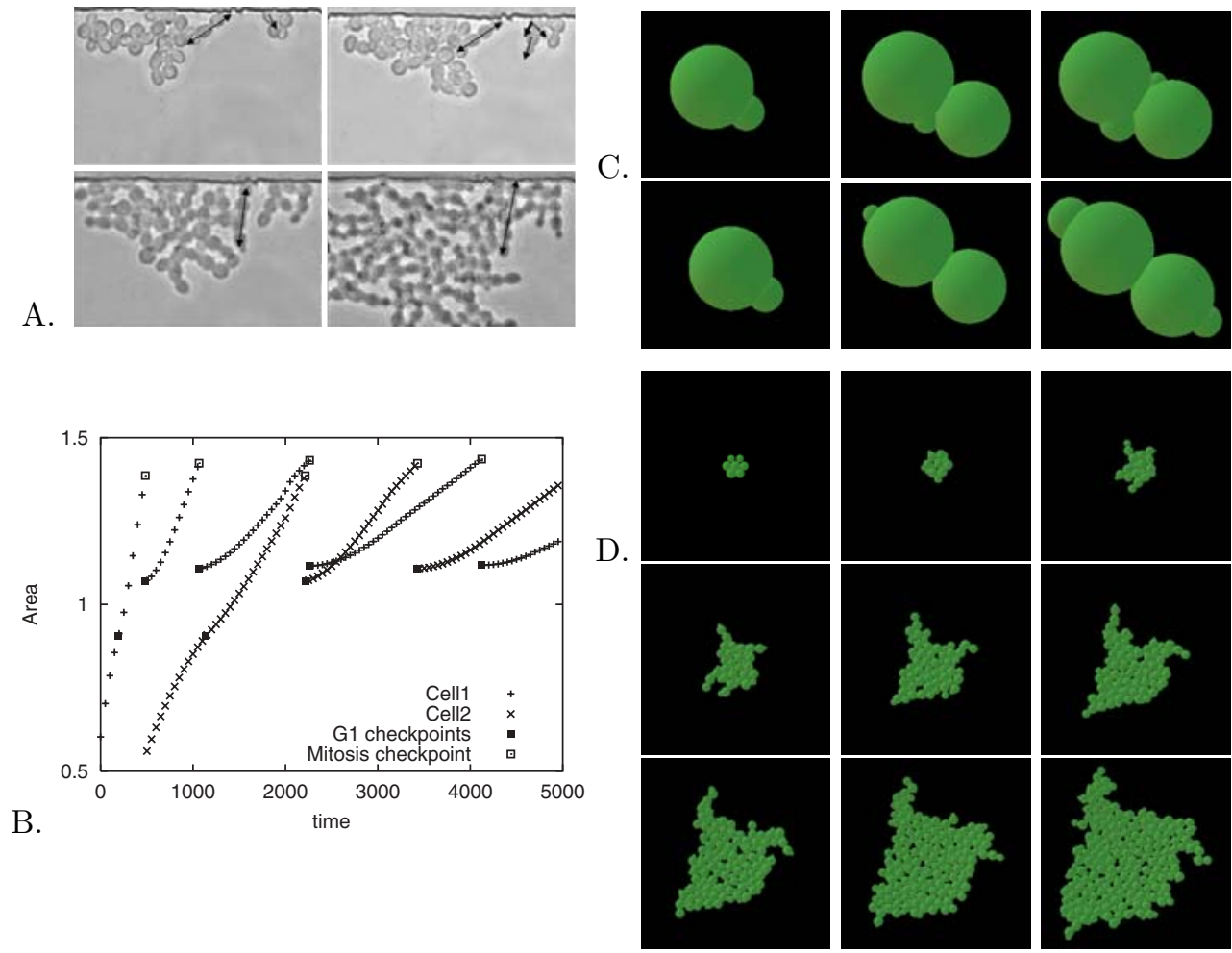

FIG. 2.1. A. Experimental data showing the bipolar growth of diploid C. albicans. Single-pointed arrows show the direction of cell division (from mother to daughter). In the upper right panel the direction of the previous division is indicated by a thicker arrow. The double-pointed arrow indicates an almost linear chain of cells resulting from unipolar cell division. B. Plot showing time vs. size for two cells. Note that the time scale is set by the growth parameters $\left(k_{g 1}, k_{g 2}\right)$ which are set to 0.01 throughout all simulations. The size is in arbitrary units, and typically the spheres have a maximal radius $r_{\max }=0.6$. Also points where checkpoints are passed are marked. C. Illustration of simulations with axial (top row) and polar (bottom row) cell divisions. The left images show the location of a bud. In the middle image the cell has divided and a new bud is formed from the mother, and in the right image also the daughter cell has formed a bud. D. Typical time evolution of a simulation. This specific case uses polar divisions $\left(k_{\text {axialFrac }}=0\right)$, growth dependence on number of neighbors $\left(k_{n e i g h F a c}=3\right)$, and cell adhesion $\left(2 k_{\text {cell }}^{\text {celcell }}=k_{\text {adFac }}^{\text {mother-daughter }}=0.1\right)$. The simulation starts with 7 cells in a hexagonal grid and continues until the colony reaches 300 cells.

with $\chi_{0}$, the vector corresponding to the cell division leading to the formation of the daughter cell, then this pattern of division is termed unipolar. If the $\chi_{i}$ are opposite to $\chi_{0}$, the division pattern is still referred to as axial. It is a conventional wisdom among the yeast biologists that the axial division pattern leads to "bunching" of cells into tightly packed cell spheroids, whereas unipolar and bipolar cell divisions can help expansion of a colony. The so-called dimorphic switch from axial to polar division patterns often occurs when cells find themselves starved for nutrients. Thus, if the polar pattern indeed can provide an opportunity for exploration of the cell's surroundings for food, the dimorphic switch can indeed be a good evolutionary strategy. In the following analysis we will use the term polar to describe both bipolar and unipolar division-polarity patterns.

For the pathogenic yeast Candida albicans, no haploid form is known to exist. 
Thus all the cell divisions under normal conditions can be assumed to display polar patterns (Figure 2.1A).

Another descriptor of yeast-cell division in S. cerevisiae and C. albicans is the size threshold characterizing bud formation and mother-daughter cell separation. A sufficiently large yeast cell can initiate a cell-division cycle (the so-called G1 phase of the cell cycle) by selecting a bud site and forming a membrane exvagination, or bud, that initially is just a semispherical projection present on the surface of a cell. Of importance here is that in axial division, cell division is asymmetric. That is, the daughter cell at the time of cell division is always smaller than the mother cell. As the cells switch to polar division, the division becomes more symmetric, with the size of the daughter cell approaching that of the mother cell [11].

As noted above, an additional important determinant of colony morphology is cell-cell adhesion. In fact, there are two types of cell adhesion that we will distinguish. One is mother-daughter cell adhesion, and the other is cell adhesion between cells that can colocalize by chance. The reason for this distinction is that motherdaughter cell adhesion can occur in a natural way following cell separation and that cell-cell separation is often incomplete. For instance, under the circumstances of nutrient (especially glucose) depletion, yeast cells may enter a filamentous and invasive form of growth, in which mother-daughter cell separation is limited and cells form extended chains $[3,15]$. Interestingly, the same starvation conditions can upregulate floculins, proteins responsible for a nonspecific increase in cell-cell adhesion, most notably Flo11p. Expression of these proteins has also been implicated in increased mother-daughter cell adhesion.

Finally, an important facet of yeast-cell division and colony expansion is the dependence of cell growth on the nutrient supply. In general, as yeast cells begin to starve, their growth rate diminishes until they enter into the so-called stationary phase, when cell division stops $[8,9]$. As we will consider the development of colonies close to or at the point of starvation, the accumulation of cells around a given cell can be assumed to lead to a gradual slowing of the growth rate. This assumption is consistent with the common modeling prediction of vigorous yeast-cell growth and division at the periphery of a growing colony and an almost complete absence of cell growth at the center of a colony.

\section{Model description.}

3.1. Cell growth. Cells are modeled as elastic spheres of variable size, whose positions can change during simulation. We chose to allow for a faster than linear growth for a cell up to a certain radius. The end results are not particularly sensitive to this assumption. More specifically, we have modeled the growth rate to be a function of the radius $f(r)$ multiplied by a "slowing" factor $g\left(r, r_{\max }\right)$, where $r_{\max }$ is the maximal radius of the cell. The slowing factor serves to decrease the growth rate to zero as the maximal cell size is approached and is used, e.g., in [22]. The growth is thus implemented as

$$
\frac{d r}{d t}=f(r) g\left(r, r_{\max }\right)
$$

where

$$
f(r)=k_{g 1} r+k_{g 2}
$$


and

$$
g\left(r, r_{\max }\right)=\left(1-\frac{f(r)}{f\left(r_{\max }\right)}\right) .
$$

As can be seen from Figure 2.1B, these assumptions can lead to almost linear cell growth prior to cell division.

3.2. Cell-division cycle. The cell cycle in the model is completely driven by the growth of the cells, with no underlying molecular mechanisms specifically accounted for. Two checkpoints are introduced to determine when a new bud begins to form (G1 phase) and when the mother and daughter cells separate (mitosis) as follows:

- "G1 checkpoint": $r>k_{G 1} r_{\max }$ and no other bud present on the cell;

- "mitosis checkpoint": $r_{\text {bud }}>k_{\text {mitosis }} r_{\text {max }}$.

In Figure 2.1C we show how the cell cycle is visualized in the model. In the particular case shown, the division-polarity pattern is bipolar with the second bud on the mother cell being formed at the pole opposite the site of the first bud. The first bud becomes a separate cell and continues to grow before it too can form a bud, thus becoming a mother cell.

3.3. Cell-division polarity. A cell polarity is defined for each cell, and new buds are formed according to this polarity. A spatial axis vector, $\chi_{\text {cell }}$, is defined as being in the direction between the mother and daughter at a division, and the next bud is formed in either a direction almost colinear to the vector or the opposite direction. Axial and polar divisions are defined in the model. If the direction is defined from the cell positions at a division $\boldsymbol{\chi}=\left(\boldsymbol{x}_{\text {daughter }}-\boldsymbol{x}_{\text {mother }}\right)$, then axial polarization is defined by new buds forming close to

$$
\chi_{\text {mother }}=\chi
$$

and

$$
\chi_{\text {daughter }}=-\chi,
$$

leading to the new buds forming close to the old mother-daughter connection point. The bipolar division pattern is defined by having the new buds form as follows:

$$
\chi_{\text {daughter }}=\chi
$$

and

$$
\chi_{\text {mother }}=-\chi
$$

In the model, the new buds on the daughter and mother cell always form away from the old scar. The implementation has a parameter, $k_{\text {axialFrac }}$, which sets the fraction of divisions that are axial and the fraction that are polar $\left(1-k_{\text {axialFrac }}\right)$.

3.4. Mechanical interactions. The mechanical interactions are implemented as spring forces between the centers of the spheres. The cells represent a highly viscous medium with friction [20], and, in the model, velocity (not acceleration) is proportional to force. Also an adhesion force is allowed for, but this is usually a fraction of the repulsive force, since high overlap between cells represents a highly unphysical state. Some overlap is allowed for and cells should be interpreted as having a "squeezed" 
shared wall. The contribution from a neighboring cell pair to the positional update is a two-dimensional extension of the one-dimensional case described by

$$
{\frac{d x_{i}}{d t}}_{\text {repulsive }}=k_{\text {spring }}\left(d-d_{\text {relax }}\right)=k_{\text {spring }}\left(\left(x_{j}-x_{i}\right)-k_{r F r a c}\left(r_{i}+r_{j}\right)\right)
$$

and

$$
{\frac{d x_{i}}{d t}}_{\text {attractive }}=k_{\text {spring }} k_{\text {adFrac }}\left(d-d_{\text {relax }}\right) .
$$

The parameter $k_{r F r a c}$ allows for a relaxing distance slightly different from the sum of the radii, and if the spheres are a mother-bud pair, the bud has a relaxing distance equal to $r_{\text {mother }}$. Different values of the parameter $k_{a d F r a c}$ are used if the pair is a mother-bud, a mother-daughter, or an "unrelated" cell-cell pair. In addition to this, the adhesion part is only used at short distances. The contribution is truncated if the distance between two cells, $i, j$, is larger than $k_{n e i g h b o r}\left(r_{i}+r_{j}\right)$ to allow for cell-cell separation.

3.5. Growth inhibition by immediate neighbors. To test dependence on nutrients, in some simulations the growth is made dependent on the number of immediate cell neighbors in contact with a given cell. As discussed above, it is assumed that cells with more neighbors within the colony compete more for nutrients, and hence grow slower. In the model implementation, the growth parameters $k_{g 1}$ and $k_{g 2}$ in (3.2) are changed as follows:

$$
k_{g(1,2)} \rightarrow \frac{k_{g(1,2)}}{\left(k_{n e i g h F a c} N_{n e i g h}+1\right)},
$$

where $N_{\text {neigh }}$ is the number of neighbors, and $k_{\text {neighFac }}$ is a factor setting the strength of the dependence. When $k_{n e i g h F a c}$ is zero, there is no dependence. In addition, the dependence on the number of neighbors according to the power law with the absolute exponent greater than unity was investigated (i.e., the exponent of the denominator in (3.10)). Larger regions when calculating the $N_{\text {neigh }}$ in the simulations were also examined. The results are similar, and a few of these simulations are presented later in the paper.

3.6. Different adhesion strength. In a number of simulations, different orders of magnitude of the adhesion strength are compared. In most simulations the mother-daughter adhesion is twice that of other cell-cell adhesion $\left(2 k_{\text {adFac }}^{\text {cell }- \text { cell }}=\right.$ $k_{\text {adFac }}^{\text {mother-daughter }}$ ).

3.7. Implementation. In the simulations, the colony growth starts with the same number of cells (usually 7 cells arranged in a symmetric hexagonal pattern) and are allowed to proceed until the same ending time. The model is implemented in a special-purpose $\mathrm{C}++$ code, based on code for developmental biological systems [10]. It simulates the time dynamics by integration of the differential equations using a fifthorder Runge-Kutta solver with adaptive time steps [17]. All simulations are in two dimensions. In most plots the data points represent the average of 50 simulations with similar initial conditions (Appendix B) and parameter sets. The error bars represent the corresponding errors (standard deviation divided by $\sqrt{N_{\text {runs }}}$ ). All parameter values used are described in Appendix A. Visualization of yeast-cell colonies has been done by scripts written in java3D (Figures 2.1 and 3.1) and Matlab (Figures 4.3 and 4.5). 
3.8. Metrics of colony size and morphology. To evaluate the colony morphology in a consistent manner in multiple runs with different parameters, two metrics were introduced. The first metric, referred to as the colony sparsity, $R$, is defined as the minimal colony-covering circle area divided by the colony area (Figure 3.1). It is defined to quantify the colony shape's deviation from a circle. The closer the colony shape is to a circle, the closer the value of the metric to unity. This metric estimates the degree of colony shape inhomogeneity, including internal cell-free spaces, finger-like cell outgrowths, or colony extension as a whole in a particular direction.
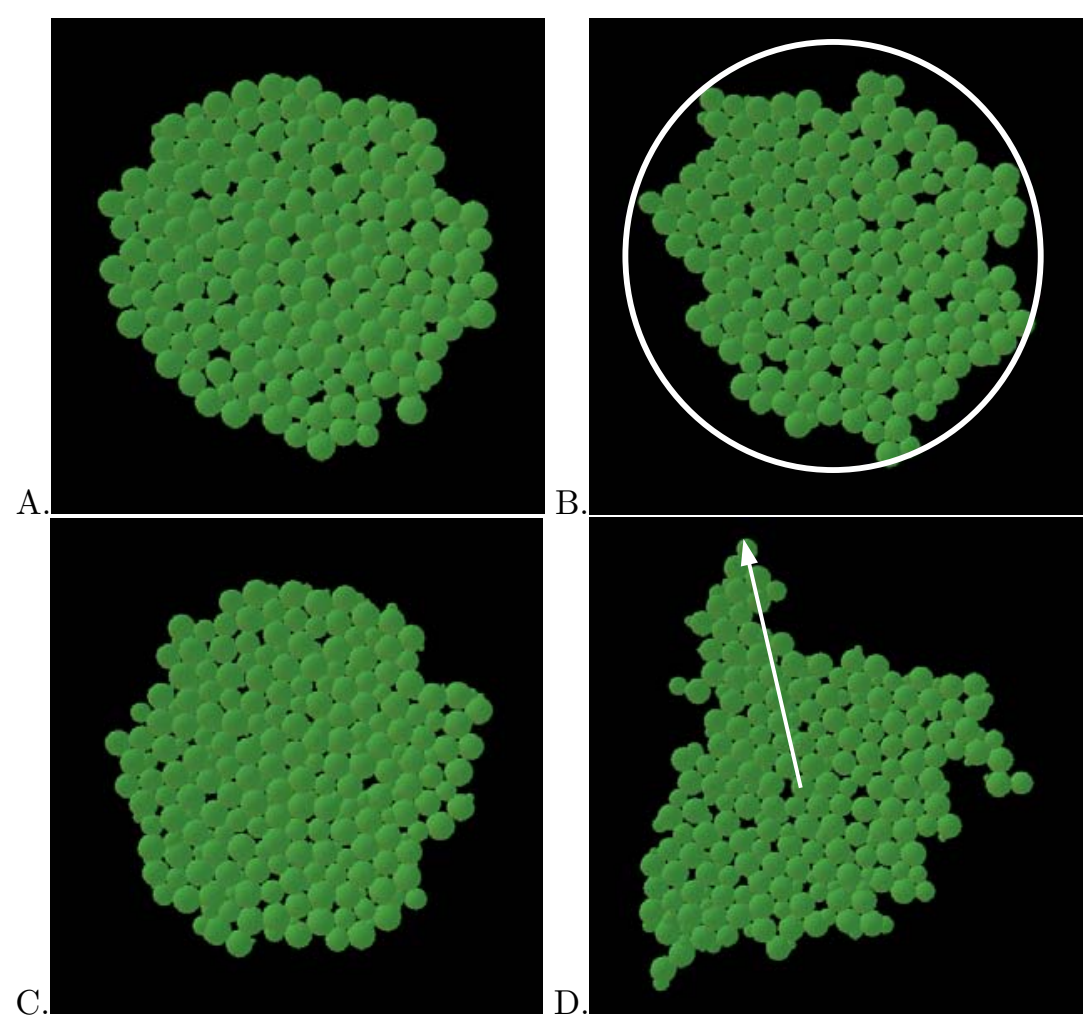

FIG. 3.1. Illustration of final colony morphologies for different parameter sets and the definition of morphology metrics. All simulations started from a similar initial configuration (Appendix B) and ended when the colony reached 300 cells. A. and B. Axial division $\left(k_{\text {axialFrac }}=1\right)$. C. and D. Polar division $\left(k_{\text {axialFrac }}=0\right)$. A. and C. No growth dependence on the number of neighbors $\left(k_{n e i g h F a c}=0\right)$. B. and D. Growth dependence on the number of neighbors $\left(k_{n e i g h F a c}=3\right)$. In all simulations $2 k_{\text {adFac }}^{\text {cell-cell }}=k_{\text {adFac }}^{\text {mother-daughter }}=0.1$. C. The colony sparsity measure, $R$, is defined as the area of the smallest circle covering the colony divided by the total cell area. D. The colony expanse measure, $R_{\max }$, is defined as the maximal distance from the colony center to any cell within the colony.

The second metric, also illustrated in Figure 3.1, referred to as the colony expanse, $R_{\max }$, is defined as the maximum distance between the colony center and any cell within the colony. The colony center is defined as the center of mass of the colony. This metric indicates how far-reaching the colony growth is, primarily away from the colony initiation point, over a certain period of time. 


\section{Results.}

4.1. The role of the axial vs. polar division polarity. First, we set out to find whether the switch from the axial to the bipolar division-polarity pattern occurring as cells begin sensing depletion of nutrients can indeed lead to a "foraging" colony behavior, in which the colony can quickly expand to locations with potentially higher supply of resources. Thus we ran multiple simulations, in which the probability of axial vs. polar division polarity $\left(k_{\text {axial Frac }}\right)$ was varied from simulation to simulation but kept constant during individual simulation runs. Surprisingly, if we assumed no dependence of the growth rates of single cells on the number of surrounding cells $\left(k_{\text {neighFac }}=0\right)$, there was no measurable effect of the division pattern on colony expansion, as measured by either of the metrics (Figure 4.1A and B). This observation was not affected by the assumed cell-cell adhesion strength. Thus, simply switching the cell-division polarity from axial to polar does not necessarily lead to any significant effect on the colony size.

To see if the effect of cell-growth inhibition observed in the state of nutrient depletion would affect colony expansion, we varied the parameter $k_{n e i g h F a c}$, trying to test the colony-size dependence on division polarity and cell-cell adhesion (Figure 4.1A-D). Unexpectedly, we have observed that with increased inhibition of cell growth and division as a function of the number of neighboring cells, the colony sparsity and expanse tend to increase. This counterintuitive increase occurs even when the division pattern is $100 \%$ axial, in which case the colony area can increase by about $20 \%$ with a corresponding increase in the expanse metric. When the division pattern is shifted to either mixed axial/polar or $100 \%$ polar, the increase in the colony sparsity and size is even more significant, displaying also a strong dependence on $k_{n e i g h F a c}$. Statistically, the effect of the division pattern is significant when the probability of polar division within a colony exceeds $1 / 2$. The expanse metric displays a higher degree of variability compared to the sparsity metric. The dependence of the averages of both metrics on the fraction of axial division in the population seems to follow a power law $\left(k_{n e i g h F a c}>1\right)$, but, due to high variability, no fit was attempted. Thus, yeast colonies can, indeed, significantly increase their size and change morphology in response to both cell-growth inhibition and a switch to polar growth patterns. Regulated growth inhibition is a necessary condition for this increase. The effect of an increase in cell-cell adhesion is somewhat more complex and will be discussed in detail below.

4.2. The role of the growth inhibition by immediate neighbors. Since the strength of growth inhibition by the immediate neighbors of a given cell, $k_{n e i g h F a c}$, is the key parameter in determining the extent of colony expansion, we studied the dependence of both colony-size metrics on this parameter in more detail (Figure $4.1 \mathrm{C}-\mathrm{F}$ and Figure 4.2). The colony expanse and sparsity exhibited positive saturating dependence on $k_{n e i g h F a c}$, with all curves tending to a limiting value. For the fully polar division pattern, the maximum increase in colony sparsity was estimated to be approximately twofold, with 80-90\% increase achieved around $k_{n e i g h F a c}=4$. Under the same conditions, the colony expanse increased by about $50 \%$. Increasing cell-cell adhesion led primarily to lower dependence on $k_{n e i g h F a c}$ at higher $k_{n e i g h F a c}$ values, with saturation setting in around $k_{n e i g h F a c}=1-2$. The colonies exhibiting fully axial division polarity exhibited the least dependence on $k_{n e i g h F a c}$. In keeping with Figure $4.1 \mathrm{~A}$ and $\mathrm{B}$, the statistical differences in the colony-size and morphology metrics occur primarily in the cases in which the fraction of polar division during colony growth is higher than $1 / 2$. 
A.
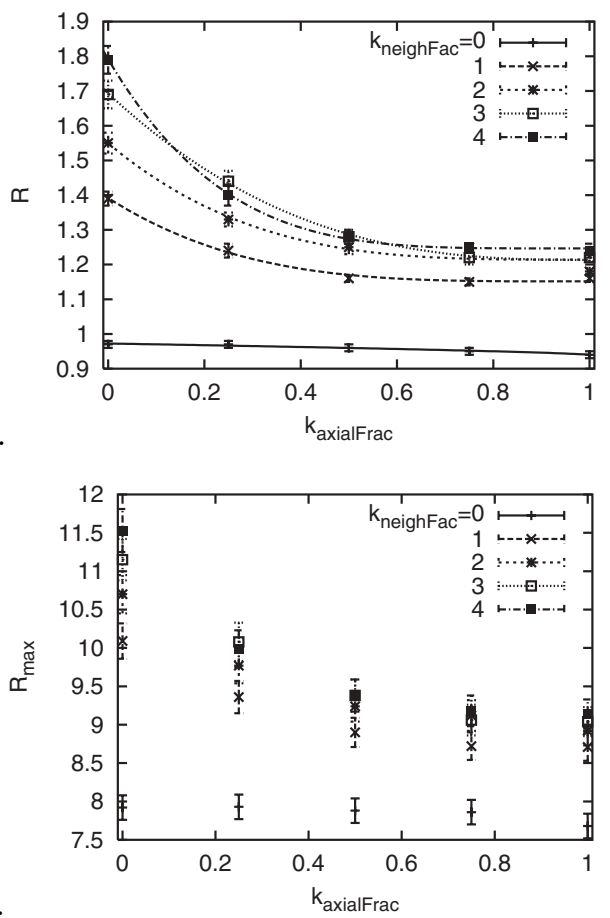

B.

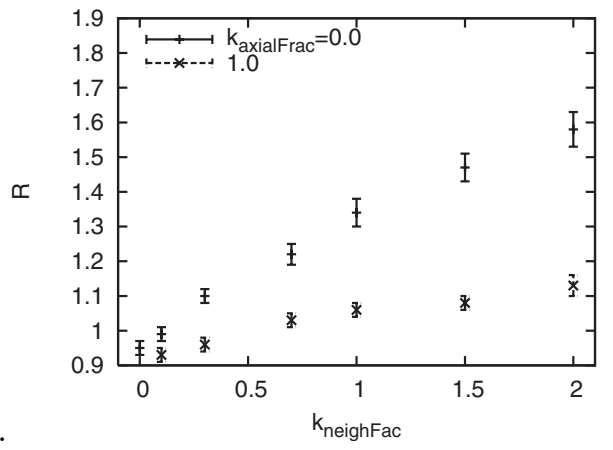

C.
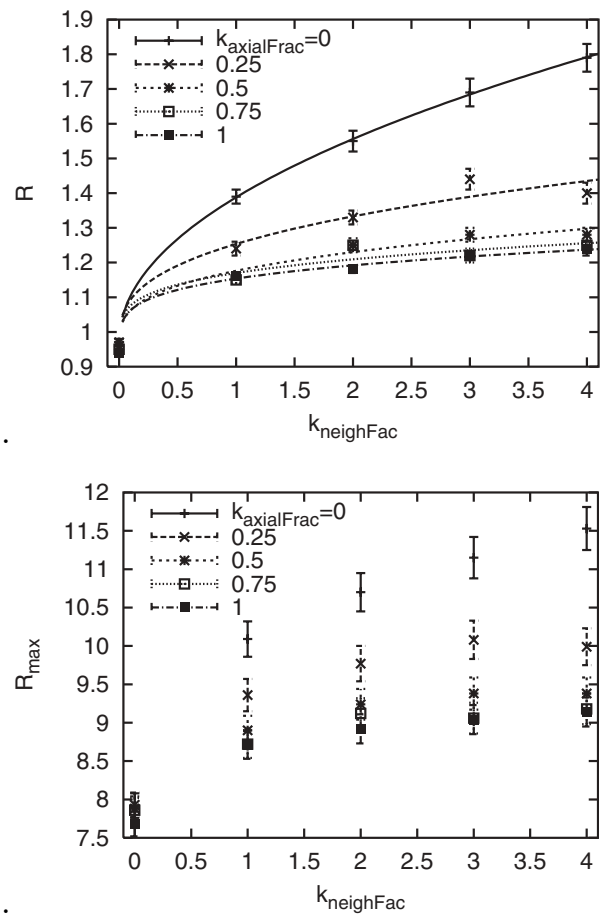

D.

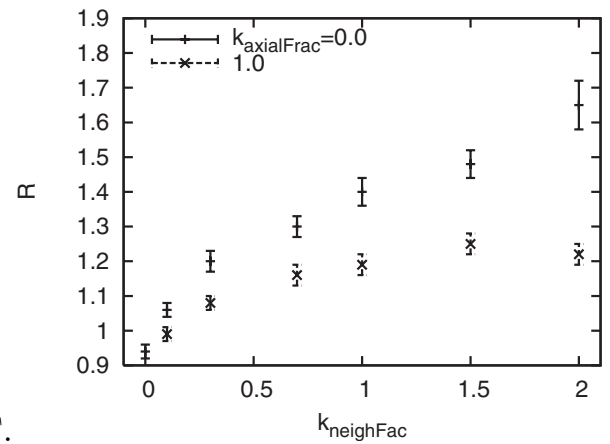

FIG. 4.1. Statistics for the sparsity measure, $R$, and the colony expanse measure, $R_{\max }$, for 50 runs per point. Each run is started at a random initial configuration (Appendix B) and is stopped when the colony reaches 300 cells. A. Fraction of axial divisions $\left(k_{\text {axialFrac }}\right)$ vs. $R$ for different values of $k_{n e i g h F a c}$. B. Fraction of axial divisions vs. $R_{\max }$ for different values of $k_{n e i g h F a c}$. C. Neighbor growth factor $\left(k_{n e i g h F a c}\right)$ vs. $R$ for different values of $k_{\text {axialFrac. }}$ D. Neighbor growth factor vs. $R_{\max }$ for different values of $k_{\text {axialFrac }}$. E. Neighbor growth factor vs. $R$ for polar and axial divisions and immediate neighbors ( $k_{\text {neighbor }}=1.1$ for calculating $\left.N_{\text {neigh }}\right)$. F. Neighbor growth factor vs. $R$ for polar and axial divisions and a larger neighbor region ( $k_{\text {neighbor }}=2.1$ for calculating $\left.N_{\text {neigh }}\right)$. A.-D. $2 k_{\text {adFac }}^{\text {cell-cell }}=k_{\text {adFac }}^{\text {mother-daughter }}=0.1$. E.-F. $2 k_{\text {adFac }}^{\text {cell-cell }}=k_{\text {adFac }}^{\text {mother-daughter }}=$ 0.05. Error bars indicate errors in averages (standard deviation divided by $\sqrt{N_{\text {runs }}}$ ).

We also varied the surrounding region for which the number of neighbors affects the growth (Figure 4.1E-F). In Figure 4.1E the immediate neighbors influence the growth, while in Figure 4.1F a larger region of cells exerts this influence. The same overall dependence patterns as those observed for immediate neighbors influencing growth are also seen for when the larger neighboring region of cells is important for 
A.
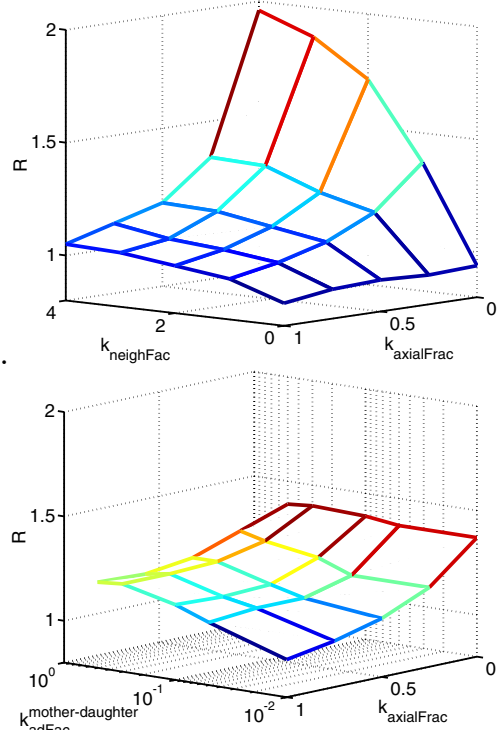

C.
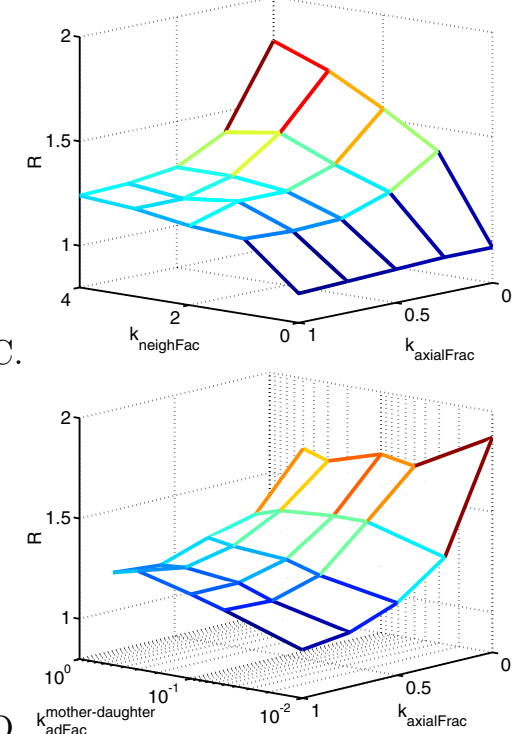

FIG. 4.2. Statistics for the sparsity measure, $R$, for 50 runs per point. Each run is started with a random initial configuration (Appendix B) and is stopped when the colony reaches 300 cells. A. and C. Fraction of axial divisions ( $\left.k_{\text {axialFrac }}\right)$ and neighbor growth factor $\left(k_{n e i g h F a c}\right)$ vs. $R$. A. $k_{\text {adFac }}^{\text {mother-daughter }}=0.01$. C. $k_{\text {adFac }}^{\text {mother-daughter }}=0.1$. B. and D. Fraction of axial divisions and mother-daughter adhesion ( $\left.k_{\text {adFac }}^{\text {mother-daughter }}\right)$ vs. R. B. $k_{n e i g h F a c}=1.0$. D. $k_{n e i g h F a c}=3.0$. In all simulations $k_{\text {adFac }}^{\text {cell-cell }}=0.5 k_{\text {adFac }}^{\text {mother-daughter }}$.

growth inhibition, although the difference between polar vs. axial division patterns somewhat decreased.

4.3. The role of cell-cell adhesion. As indicated above, cell-cell adhesion was one of the parameters varied in the simulations. To distinguish between motherdaughter cell adhesion and adhesion between cells that colocalize by chance during simulations, we considered explicitly two cases: only mother-daughter cell adhesion is variable and both mother-daughter and other cell-cell interaction are variable (Figure 4.3). When the mother-daughter adhesion $\left(k_{\text {adFac }}^{\text {mother-daughter }}\right)$ only is varied, the bipolar division pattern coupled with high growth inhibition can lead to the formation of several prominent finger-like extensions from the colony (Figure 4.3B). These extensions are connected to the rest of the colony. The exact strength of cell-cell adhesion seems to have little effect on this property. When $k_{\text {adFac }}^{\text {mother-daughter }}$ is increased with axial division polarity and strong inhibition of cell growth, the colonies tend to be asymmetric with large intracellular spaces (Figure 4.3A). When the growth inhibition is assumed to be weak, both division-polarity patterns lead to similar circular colonies (as in Figure 3.1A,C).

We also examined the case in which both $k_{\text {adFac }}^{\text {mother-daughter }}$ and "unrelated-cell" adhesion $\left(k_{a d F a c}^{\text {cell-cell }}\right)$ were variable. We still assumed that the mother-daughter adhesion was considerably stronger $\left(k_{\text {adFac }}^{\text {mother-daughter }}=2 k_{\text {adFac }}^{\text {cell }- \text { cell }}\right)$. The main distinction between the results arising from this simulation and the simulations for variable $k_{\text {adFac }}^{\text {mother-daughter }}$ is that the colonies become more compact and symmetric. More detailed analysis of the colony-growth time sequences indicates that the finger-like projections forming in the colonies with high probabilities of polar division quickly 
A.

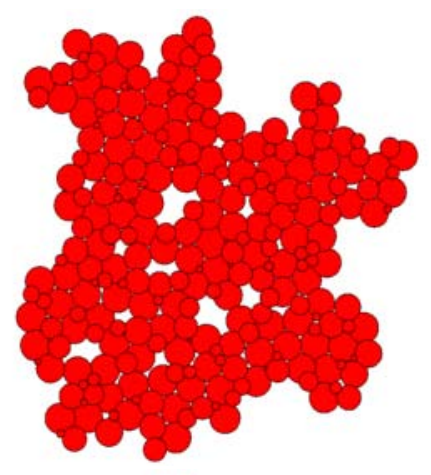

C.

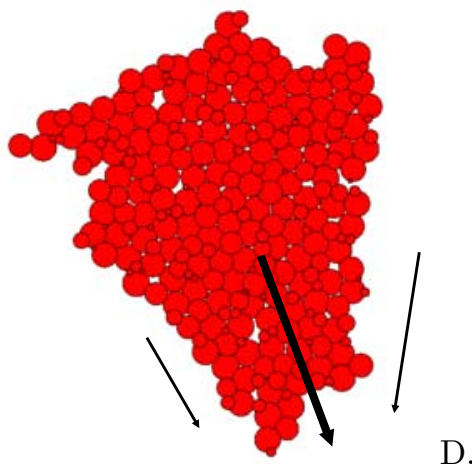

B.
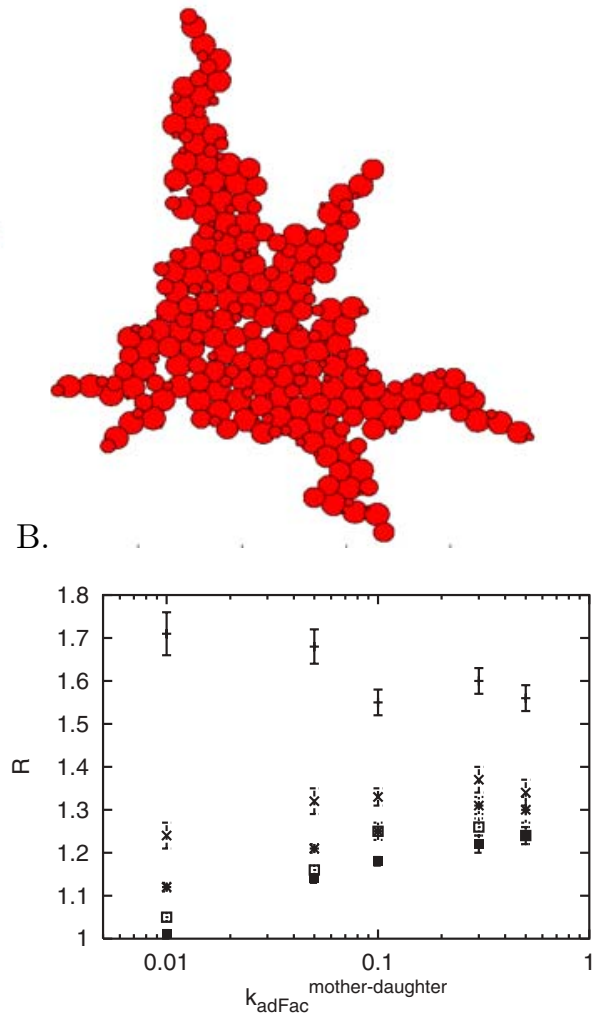

FIG. 4.3. A. An example of a colony morphology arising for high mother-daughter cell adhesion, high strength of growth inhibition, and axial division polarity $\left(k_{\text {adFac }}^{\text {mother-daughter }}=0.5\right.$, $k_{\text {adFac }}^{\text {cell-cell }}=0.1, k_{\text {axialFrac }}=1, k_{\text {neighFac }}=1$; note that in this simulation we examined stronger neighbor-growth inhibition, by squaring the denominator in (3.10)); B. Same as A. but with polar rather than axial division polarity $\left(k_{\text {axialFrac }}=0\right)$; C. Same as B. but with an increase in cell adhesion between neighboring cells (not necessarily mother-daughter pairs). The thin arrows indicate merging of two separate finger-like growth extensions into a single, large colony extension, whose growth direction is indicated with the thick arrow $\left(k_{\text {adFac }}^{\text {mother-daughter }}=2 k_{\text {adFac }}^{\text {cell-cell }}=0.5\right)$. D. Statistics of the sparsity measure, $R$, for 50 runs per point. Each run is started at a random initial configuration (Appendix B) and is stopped when the colony reaches 300 cells. The plot shows mother-daughter cell adhesion $k_{a d F a c}^{\text {mother-daughter }} v$ v. $R$ for different fractions of axial divisions $\left(k_{\text {axialFrac }}=0(+), 0.25(\times), 0.5(*), 0.75(\square)\right.$, and $\left.1(\mathbf{\square})\right) . k_{\text {adFac }}^{\text {cell }- \text { cell }}=0.5 k_{\text {adFac }}^{\text {mother-daughter }}$ and $k_{n e i g h F a c}=2$.

merge either with each other or with the main body of the colony (Figures 2.1D and 4.3C). Merging of the finger-like extensions often leads to coorientation of cell-division patterns on at least one side of the colony resulting in colony extension in a certain direction. We term this behavior the "zipper effect." This effect can potentially explain large-scale oriented growth in yeast macrocolonies, leading to a "spaghetti" pattern visible to a naked eye.

Another interesting effect of increasing cell-cell adhesion is the dependence of the colony sparsity on adhesion strength and growth inhibition. If growth is not inhibited by immediately adjacent cells, the sparsity of the colony does not depend on whether the division is axial or polar, and the sparsity increases with increasing adhesion. Interestingly, at higher neighbor growth-inhibition strengths, the sparsity of the 
colonies of axially dividing cells increases, whereas the sparsity of the polarly dividing cells decreases with increasing cell-adhesion strength (Figures 4.3D and 4.2B,D). The colonies with mixed division patterns show intermediate dependence that may be relatively flat. Thus cell-cell adhesion leads to compaction of colonies with polar division polarity, whereas the colonies with prevalently axial division may assume less well packed yet still relatively symmetric morphologies. The decreased sparsity of "polarly" dividing colonies is likely to be due to the "zipper" effect discussed above.

4.4. A comment on mother-daughter size asymmetry. As mentioned in section 2 the asymmetry of the mother-bud pair at mitosis can differ, with the axial division pattern being more asymmetric than the polar one. In the model this asymmetry is tuned by the parameter $k_{\text {mitosis }}$, defining the mitosis checkpoint constraint. The result of varying this parameter is shown in Figure 4.4. The effect on the development of colonies with axial division pattern is minor. In the case of the polar division pattern, there is an increased colony sparsity concomitant with an increased colony symmetry, although the effect is again dependent on an inhibition of growth by the neighboring cells. For both axial and polar division patterns there is a tendency towards decreased sparsity when the bud size approaches the maximal cell size due to a decreased growth (more specifically, due to a decreasing factor in (3.1)). We have consistently used the value of 0.7 for $k_{m i t o s i s}$ in all other simulations in this paper.

A.

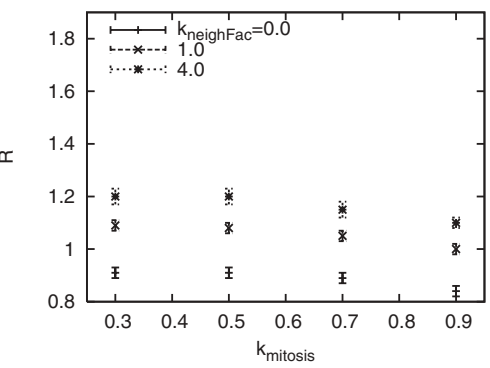

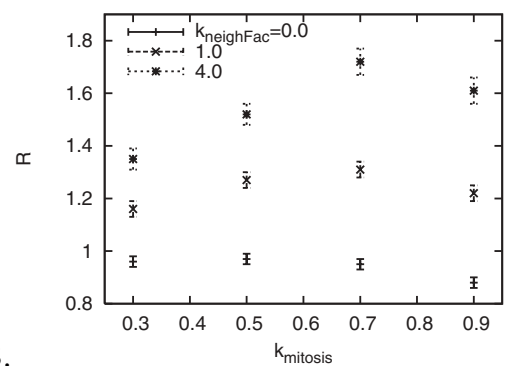

FIG. 4.4. Dependence on mother-bud asymmetry. Statistics for the sparsity measure, $R$, as a function of $k_{\text {mitosis }}$ for different values of $k_{\text {neighFac. }}$. Each point is the average of 50 runs from random initial conditions (Appendix B). A. axial division pattern, $k_{\text {axialFrac }}=1$. B. polar division pattern, $k_{\text {axialFrac }}=0$. In all simulations $2 k_{\text {adFac }}^{\text {cell }- \text { cell }}=k_{\text {adFac }}^{\text {mother-daughter }}=0.05$.

4.5. The dynamic switching of parameters during colony growth. In the simulations described in sections $4.1-4.3$ we have been primarily interested in the behavior of a growing colony with parameter values fixed throughout each simulation. Implicitly, it was assumed that the parameters are fully determined by the conditions of the growth medium, such as the concentration of nutrients. In most cases this assumption is probably valid. However, it might be possible that, as a colony grows, the nutrients can be depleted and the colony can switch in a synchronous fashion its division pattern as well as the level of cell adhesion and growth inhibition by neighbors. Such synchronous changes in macrocolony behavior have been reported previously [16]. It is thus of interest to see what behavior can be expected in a colony that begins growing in an axial pattern and then gradually shifts to a fully polar pattern with increasing growth suppression by the immediate neighbors. The time course of such a simulation is shown in Figure 4.5. As the colony expands, it initially grows symmetrically, gradually slows down, and undergoes apparent growth-front instability ultimately leading to the formation of finger-like extensions, some of which merge. 
A.
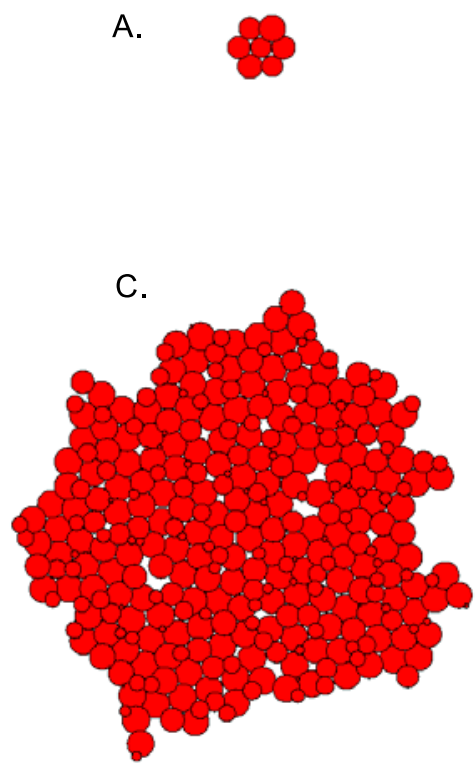

B.

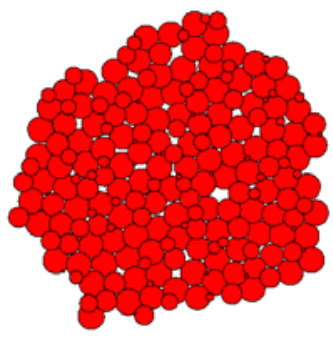

D.

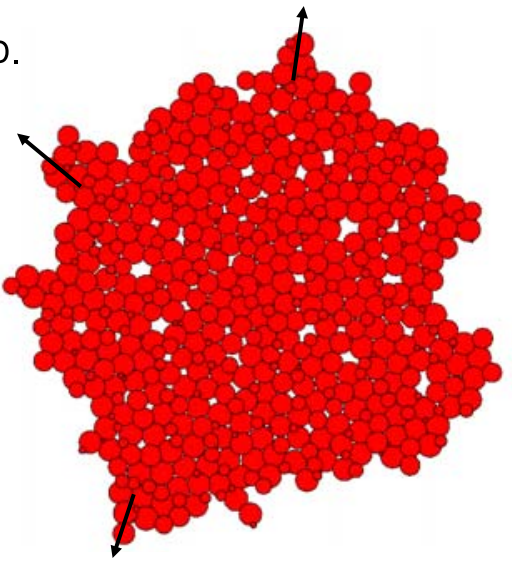

FIG. 4.5. Equally spaced time frames showing the development of a colony with a gradually increasing fraction of cells undergoing polar division and increasing sensitivity to the immediate growth inhibition. The arrows indicate the preferred directions of the colony growth through formation of colony extensions. The simulation is divided into one part with $k_{\text {axialFrac }}=1$ and $k_{n e i g h F a c}=0$, then a part with linearly decreasing $k_{\text {axialFrac }}$ and linearly increasing $k_{n e i g h F a c}$, and finally a part with $k_{\text {axialFrac }}=0$ and $k_{n e i g h F a c}=4$. When 600 cells are reached, the simulation stops. In this particular simulation, also a maximal number of 4 daughters are allowed for each cell $\left(k_{\text {adFac }}^{\text {mother-daughter }}=2 k_{\text {adFac }}^{\text {cell-cell }}=0.1\right)$.

The ensuing directed growth can potentially help the progeny of cells in the colony escape into a nutrient-rich environment. Thus, even in an initially large, symmetric colony, a switch to polar division can ultimately lead to asymmetric colony extension.

5. Discussion. This study presents a simple model of yeast microcolony growth under normal and partially starved conditions. This model was called upon to support or refute the common assumption made in the field of yeast biology, namely, that a switch in the polarity of cell division from axial to polar is responsible for the ability of a colony to exhibit rapid expansion to regions of potentially higher nutrient supply. In addition, we tried to determine whether single-cell-based simulations can yield important insights for coarser simulations involving groups of cells as elementary units or agents in a cellular-automaton-like simulation.

The model proposed captures many essential characteristics of yeast colony growth, providing an indication of how simple and nonmotile single-cell organisms can cooperate to develop complex morphologies and indirect locomotion. By considering the mechanics of cell adhesion, growth, and division, we observed the emergence of coordinated displacement patterns that would be difficult to predict theoretically. This model can be further extended to include intracellular molecular processes, such 
as signaling interactions and a more precise mechanism of cell-cycle control. Importantly, we have identified several parameters, whose significance in establishing nontrivial microcolony patterns can be taken into account in a coarser colony analysis, where microcolonies rather than single cells will be treated as the elementary building blocks.

The results of the study suggest several interesting and counterintuitive predictions. First, we find that merely switching the cell-division polarity does not result in appreciable differences in colony development. Rather, an additional important assumption needs to be made. Pronounced foraging behavior is primarily a result of both the polarity and growth inhibition of the cells as a function of the number of cells surrounding them. The latter assumption, the most critical one, is supported by various experimental results suggesting that, under nutrient limitation, cells in the interior regions of a colony divide very infrequently. Thus, limiting the rate of cell division can counterintuitively result in an increased colony expansion. In addition, the morphology of the colony is predicted to change, leading to a sparse organization with substantial intercellular spaces or increased elongation of the whole colony.

The findings presented here indicate that some macroscopic colony features, such as large-scale directed cell extensions in an expanding macrocolony, can be modeled with an approximation of a set of yeast-cell blocks each representing the microcolonies modeled here. Since the assumption of polar division and neighboring-cell growth inhibition are the critical parameters determining the foraging behavior of microcolonies, a larger-scale model whose behavior is based primarily on cell-cell adhesion can be decoupled from the smaller-scale model considered here. In addition, although not considered here, the model presented can be supplemented by detailed simulations of intracellular, molecular-level events. Thus a truly multiscale simulation of yeastcolony development may be within our reach.

\section{Appendix A. Parameters.}

$k_{g 1}=0.01, k_{g 2}=0.01$

The two growth parameters set the time scale and are never altered in simulations. The linear term is not necessary for the results but allows us to create buds of minimal size as spheres which grow at a nonnegligible rate.

$$
r_{\text {max }} \in\left[0.99 r_{\text {ave }}: 1.01 r_{\text {ave }}\right], \text { where } r_{\text {ave }}=0.6
$$

At initiation of simulations, cells are given random maximal radii. This assumption is not necessary for the result but causes cells to divide at more random times. The parameter is inherited by the daughters of a cell.

$$
k_{G 1}=0.9
$$

A cell must reach nearly full size to start producing a bud.

$$
k_{\text {mitosis }}=0.7
$$

When the bud reaches a threshold size the cell can divide.

$$
k_{\text {spring }}=1
$$

This is the strength of the spring force handling the positional dynamics. It is not changed in the simulations, but must be tuned in relation to the growth rate, to allow the spring to push cells away from each other.

$$
k_{\text {neighbor }}=1.1
$$

This tunes the length of the adhesion force and also defines what cells are considered neighbors. To produce adhesion it should be set larger than $k_{r F r a c}^{\text {cell-cell }}$. Also, the cells tend to arrange themselves in a closely packed pattern when spring forces are acting, so the parameter should not include next-nearest neighbors. (For a hexagonal twodimensional packing of identical circles this requirement amounts to $k_{\text {neighbor }}<\sqrt{3}$ ). 


$$
k_{r F r a c}^{\text {mother-bud }}=\frac{r_{\text {mother }}}{r_{\text {mother }}+r_{\text {bud }}}
$$

Since a cell with a bud is implemented as two spheres, there is also a spring force assumed between them. The relaxing distance is set so that the bud center is on the border of the mother sphere.

$$
k_{\text {rFrac }}^{\text {cell-cell }}=0.9
$$

The cells are not infinitely hard spheres, and some amount of overlap is allowed, reflecting some softness of the walls of closely packed cells.

$$
k_{\text {adFac }}^{\text {mother-bud }}=1
$$

Since a cell with a bud is implemented as two spheres, there is also a spring force assumed between them. The two spheres represent a single cell, and hence the bud position should not tend to move away from the mother.

$$
k_{\text {adFac }}^{\text {mother-daughter }} \in[0.01: 0.5]
$$

The adhesion of cells is set to a lower value than the repulsive force.

$$
k_{\text {adFac }}^{\text {cell-cell }} \in[0.01: 0.5]
$$

Always set to half of $k_{a d F a c}^{\text {mother-daughter }}$.

$k_{\text {axialFraction }} \in[0: 1]$

This is the fraction of divisions that are axial in the simulation.

Appendix B. Initial conditions. The initial state for the simulations usually looks like the first panel in Figure 2.1D (7 cells arranged in a symmetric hexagonal pattern), but the exact number of cells and initial cell positions does not influence the result. Each simulation is randomly initialized, with each cell given a random radius, $r \in\left[r_{\text {ave }} / \sqrt{2}: r_{\text {ave }}\right]$, a random maximal radius, $r_{\text {max }} \in\left[0.99 r_{\text {ave }}: 1.01 r_{\text {ave }}\right]$ $\left(r_{\text {ave }}=0.6\right)$, and a randomly pointing polarization vector $\chi$.

\section{REFERENCES}

[1] G. H. Braus, O. Grundmann, S. Bruckner, and H.-U. Mosch, Amino acid starvation and Gcn $4 p$ regulate adhesive growth and FLO11 gene expression in Saccharomyces cerevisiae, Mol. Biol. Cell, 14 (2003), pp. 4272-4284.

[2] J. Chant, Cell polarity in yeast, Annual Review of Cell and Developmental Biology, 15 (1999), pp. 365-391.

[3] P. J. Cullen and G. F. Sprague, Jr., Glucose depletion causes haploid invasive growth in yeast, Proc. Natl. Acad. Sci. USA, 97 (2000), pp. 13619-13624.

[4] L. J. Douglas, Candida biofilms and their role in infection, Trends Microbiol., 11 (2003), pp. 30-36.

[5] D. G. Drubin and W. J. Nelson, Origins of cell polarity, Cell, 84 (1996), pp. 335-344.

[6] P. Eggimann, J. Garbino, and D. Pittet, Epidemiology of Candida species infections in critically ill non-immunosuppressed patients, Lancet Infect. Dis., 3 (2003), pp. 685-702.

[7] J. E. HAMER AND N. J. TALBOT, Infection-related development in the rice blast fungus Magnaporthe grisea, Curr. Opin. Microbiol., 1 (1998), pp. 693-697.

[8] M. N. JaGadish AND B. L. CARTER, Genetic control of cell division in yeast cultured at different growth rates, Nature, 269 (1977), pp. 145-147.

[9] G. C. Johnston, J. R. Pringle, and L. H. Hartwell, Coordination of growth with cell division in the yeast Saccharomyces cerevisiae, Exp. Cell Res., 105 (1977), pp. 79-98.

[10] H. Jönsson, B. E. Shapiro, E. M. Meyerowitz, and E. Muolsness, Signalling in multicellular models of plant development, in On Growth, Form and Computers, S. Kumar and P. Bentley, eds., Academic Press, London, 2003, pp. 156-161.

[11] S. J. Kron, C. A. Styles, And G. R. Fink, Symmetric cell division in pseudohyphae of the yeast Saccharomyces cerevisiae, Mol. Biol. Cell, 5 (1994), pp. 1003-1022.

[12] M. Kuthan, F. Devaux, B. Janderova, I. Slaninova, C. JACQ, And Z. Palkova, Domestication of wild Saccharomyces cerevisiae is accompanied by changes in gene expression and colony morphology, Mol. Microbiol., 47 (2003), pp. 745-754.

[13] J. M. Lopez AND H. J. Jensen, Generic model of morphological changes in growing colonies of fungi, Phys. Rev. E, 65 (2002), 021903. 
[14] L. Ni And M. SNyder, A genomic study of the bipolar bud site selection pattern in Saccharomyces cerevisiae, Mol. Biol. Cell, 12 (2001), pp. 2147-2170.

[15] S. P. Palecek, A. S. PARikh, ANd S. J. Kron, Sensing, signalling and integrating physical processes during Saccharomyces cerevisiae invasive and filamentous growth, Microbiology, 148 (2002), pp. 893-907.

[16] Z. Palkova AND J. Forstova, Yeast colonies synchronise their growth and development, Cell Sci., 113 (2000), pp. 1923-1928.

[17] W. H. Press, S. A. Teukolsky, W. T. Vetterling, and B. P. Flannery, Numerical Recipes in C: The Art of Scientific Computing, Cambridge University Press, New York, 1992.

[18] T. B. Reynolds And G. R. Fink, Bakers' yeast, a model for fungal biofilm formation, Science, 291 (2001), pp. 878-881.

[19] T. S. Sams, K. Sneppen, M. H. Jensen, C. Ellegaard, B. E. Christensen, and U. Thrane, Morphological instabilities in a growing yeast colony: Experiment and theory, Phys. Rev. Lett., 79 (1997), pp. 313-316.

[20] A. E. Smith, Z. Zhang, C. R. Thomas, K. E. Moxham, and A. P. J. Middelberg, The mechanical properties of Saccharomyces cerevisiae, Proc. Natl. Acad. Sci. USA, 97 (2000), pp. 9871-9874.

[21] N. J. TALвот, Having a blast: Exploring the pathogenicity of Magnaporthe grisea, Trends Microbiol., 3 (1995), pp. 9-16.

[22] J. J. Tyson AND B. NOVAK, Regulation of the eukaryotic cell cycle: Molecular antagonism, hysteresis, and irreversible transitions, J. Theor. Biol., 210 (2001), pp. 249-263. 